\title{
Correlation of Densification Kinetics of Polycrystalline Ceramics with Colloid/Interface Variables
}

\author{
Hyun M. JANG and Jong H. MOON ${ }^{\dagger}$ \\ Department of Materials Science and Engineering, and Advanced Ceramics Processing Science Laboratory, \\ Pohang University of Science and Technology (POSTECH), Pohang 790-600, Republic of Korea
}

\section{多結晶セラミックスの緻密化速度とコロイドの安定性の関係}

Hyun M. Jang and Jong H. Moon ${ }^{\dagger}$

Department of Materials Science and Engineering, and Advanced Ceramics Processing Science Laboratory, Pohang University of Science and Technology (POSTECH), Pohang 790-600, Republic of Korea

[Received February 23, 1994; Accepted April 5, 1994]

\begin{abstract}
Densification kinetics of $\mathrm{Al}_{2} \mathrm{O}_{3}$ and $\mathrm{Y}$-doped $\mathrm{ZrO}_{2}$ ceramics was quantitatively correlated with the kinetic stability of the corresponding colloidal suspensions. Analysis of the densification data showed that increasing the kinetic stability of suspension significantly enhances the rate constant of sintering in the linear densification regime. A concept of critical stability of colloidal suspension was applied to the interpretation of densification kinetics of $\mathrm{Al}_{2} \mathrm{O}_{3}$ and $\mathrm{Y}$-doped $\mathrm{ZrO}_{2}$, and this demonstrated a direct linear correlation of the stability ratio $(W)$ with the reciprocal densificationrate constant with a transition behavior at the critical stability point.
\end{abstract}

Key-words: Densification kinetics, Stability ratio, Zeta potential, Critical stability, Second-generation pore

\section{Introduction}

The importance of uniform pore-size distribution prior to sintering is well recognized in ceramic systems. ${ }^{1)-6)}$ Ceramic powders mostly contain agglomerates that cause broad pore-size distributions in green compacts. During sintering, small pores shrink faster than large ones which exist between the agglomerates, leaving large pores at the final stage of sintering. According to Lange, ${ }^{5)}$ the coordination number of a pore (the number of grains surrounding a pore) essentially determines the poreshrinkage rate. Therefore, the large second-generation pores ${ }^{3)}$ (originated from the interagglomerate pores) are difficult to remove by sintering due to large coordination number. In view of this, extensive efforts have been made to increase the homogeneity of green compact with a minimal amount of large interagglomerate pores. Among these, colloidal consolidation route using a kinetically stable slip with a narrow particle size distribution has received increasing attention. 4),7),8)

In this study we have quantitatively analyzed effects of colloid/interface variables on the densifica-

\footnotetext{
† Now with National Institute for Research in Inorganic Materials, 1-1, Namiki, Tsukuba-shi, Ibaraki 305
}

tion kinetics (pore removal, grain growth) using $\mathrm{Al}_{2} \mathrm{O}_{3}$ and $\mathrm{Y}_{2} \mathrm{O}_{3}$-doped $\mathrm{ZrO}_{2}$ as model systems. Throughout the work reported here, we have emphasized the effects of the stability of colloidal suspension on the densification kinetics (pore removal) of a porous polycrystalline specimen.

\section{Experimental procedures}

The $\mathrm{Al}_{2} \mathrm{O}_{3}$ powder used in this study was $\alpha-\mathrm{Al}_{2} \mathrm{O}_{3}$ (Sumitomo AKP-HP), and the mean diameter of primary particles was approximately $0.45 \mu \mathrm{m}$ with a narrow size distribution. The mean diameter of $\mathrm{ZrO}_{2}$ powder (primary particle) used in the present study (High Purity Chemical Co., Japan; containing 1 wt $\%$ $\mathrm{HfO}_{2}$ ) is approximately $50-100 \mathrm{~nm}$. Three mol\% $\mathrm{Y}_{2} \mathrm{O}_{3}$ was uniformly incorporated into $\mathrm{ZrO}_{2}$ by a selective surface-induced coating ${ }^{9}$ ) of the precursor for yttria on the ultrafine $\mathrm{ZrO}_{2}$ primary particles. Figure 1 shows $\mathrm{ZrO}_{2}$ powder coated with patchwise nanometer-scale precipitates of yttrium hydroxide. The Y-doped $\mathrm{ZrO}_{2}$ powder was calcined at $700^{\circ} \mathrm{C}$ (slightly above the temperature of the eutectoid decomposition) for $2 \mathrm{~h}$. The calcined powder was mainly composed of tetragonal and cubic phases

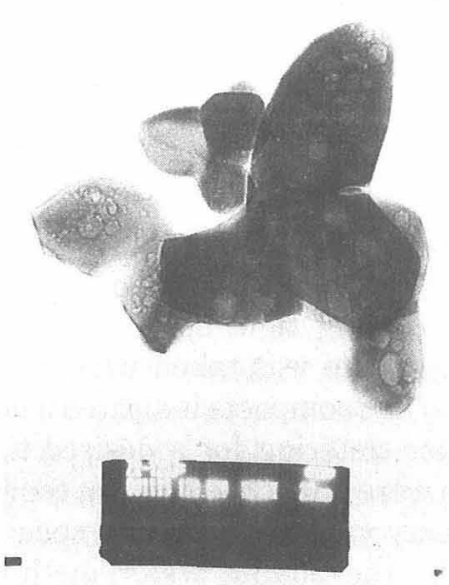

Fig. 1. Transmission electron microscopic image of zirconia powder coated with ultrafine precursor precipitates for yttria. 
with less than $5 \mathrm{~mol} \%$ of monoclinic phase.

The stability ratio $(W)$, microelectrophoresis and green density were used to characterize colloid/interface variables of $\mathrm{Al}_{2} \mathrm{O}_{3}$ and $\mathrm{ZrO}_{2}$ suspensions. Electrophoretic mobility measurements were conducted using a Lazer Zee Meter (Model 501 Pen Kem Inc., U.S.A.) by measuring the group velocity of particles under a given applied electric field. The mobility was cross-checked with the result obtained by the electrophoretic Doppler light scattering experiment using a laser light scattering device (Zetasizer III, Malvern Instrument Ltd., England). The zeta potential as a function of suspension $\mathrm{pH}$ was estimated from the data of electrophoretic mobility and ionic conductivity (ionic strength) using the method of Wiersema et al. ${ }^{10)}$ The stability ratio $(W)$ is a measure of the overall kinetic stability of a given colloid suspension. ${ }^{11)} W=1$ corresponds to the Smoluchowski's rapid coagulation limit in which the potential energy barrier for two approaching colloid particles becomes zero. The stability ratio of colloidal suspension was determined by estimating the rate constant of the initial stage of coagulation between colloid particles as a function of suspension $\mathrm{pH}$. The rate constant of coagulation, in turn, was determined by measuring change in the absorbance of colloidal suspension as a function of time using a UV-visible spectrophotometer (Model Lambda 15, Perkin-Elmer Co., U.S. A.). The rate of decrease of the turbidity at a fixed wavelength ( $450 \mathrm{~nm}$ for $\mathrm{Al}_{2} \mathrm{O}_{3}$ and 700 $\mathrm{nm}$ for $\mathrm{ZrO}_{2}$ ) was used as a measure of the rate of coagulation in this study. More detailed descriptions of experimental procedures and principles used in the determination of the stability ratio were described elsewhere. ${ }^{9)}$

Green bodies prepared by centrifugal casting (2000 rpm for $40 \mathrm{~min}$ ) of colloidal suspensions were dried at $100^{\circ} \mathrm{C}$ for $48 \mathrm{~h}$, and parts of green compacts were investigated for the pore-size distribution using a mercury porosimeter (Autopore II 9220, Micromeritics Instrument Co., U.S.A.). For the measurements, $1.5 \mathrm{~g}$ of the compact divided into 20 pieces was used. The pressure was increased in 220 steps to $410 \mathrm{MPa}$, the final pressure corresponding to a pore diameter of 3 to $4 \mathrm{~nm}$. To calculate the pore-size distribution, a mercury surface tension of $480 \mathrm{dyn} / \mathrm{cm}$ and a contact angle of $130^{\circ}$ were assumed.

The remaining green compacts were fired in air at $1480^{\circ} \mathrm{C}\left(\mathrm{Al}_{2} \mathrm{O}_{3}\right)$ and $1550^{\circ} \mathrm{C}$ (Y-doped $\mathrm{ZrO}_{2}$ ) as a function of sintering time using a heating rate of $10^{\circ} \mathrm{C} / \mathrm{min}$. Zero time was taken when a thermocouple placed near the compacts indicated a desired temperature. After sintering for a desired time period, the compacts were quenched to room temperature in air. Bulk density values of the fired specimens were determined by the boiling water method (ASTM Standard C20). The bulk density of a specimen is defined as the quotient of its dry weight divided by the exterior volume, including pores. The grain sizes of sintered $\mathrm{Al}_{2} \mathrm{O}_{3}$ specimens were determined by measuring $\sim 600$ grain-intercept lengths on scanning electron micrographs of thermally etched (1h at $1400^{\circ} \mathrm{C}$ ) polished sections. After correcting for magnification, the average intercept size was multiplied by a statistical factor (1.56) to obtain the average grain size. ${ }^{12)}$

\section{Results and discussion}

3.1 Effects of colloid/interface variables on densification and microstructure development

Figure 2 shows scanning electron micrographs of $\mathrm{Al}_{2} \mathrm{O}_{3}$ specimens sintered at $1400^{\circ} \mathrm{C}$ for $0 \mathrm{~h}$. The sample shown in Fig. 2(a) was prepared using a kinetically stable slip $(\zeta=56 \mathrm{mV}, \log W=1.58, \mathrm{pH} 3)$ and is in the intermediate stage of sintering without forming large interagglomerate pores. On the other hand, the specimen prepared under the condition of low kinetic stability ( $\zeta=4 \mathrm{mV}, \log W=0, \mathrm{pH} 9)$ shows large pores generated by a bridging between agglomerate units and is in the early stage of sintering.
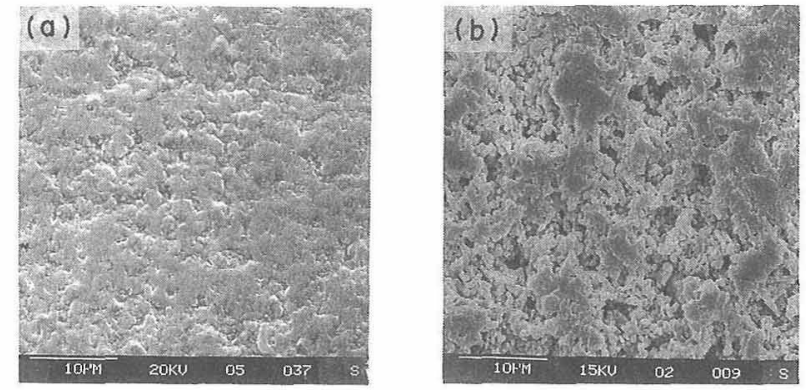

Fig. 2. Scanning electron micrographs of sintered specimens of alumina prepared at (a) $\mathrm{pH} 3$ and (b) $\mathrm{pH} 9$. The compacts were sintered at $1400^{\circ} \mathrm{C}$ for $0 \mathrm{~h}$ (no holding time).
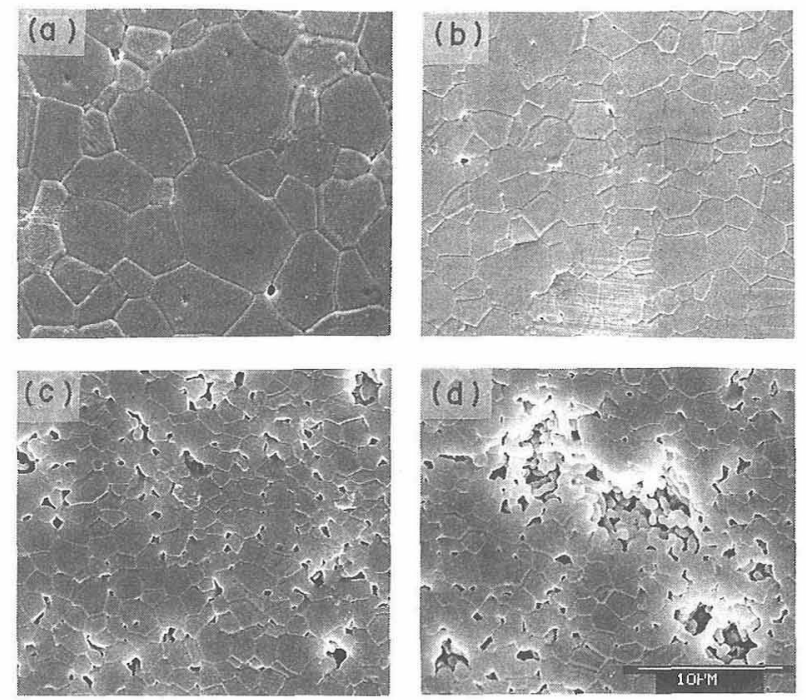

Fig. 3. Scanning electron micrographs of sintered specimens of alumina prepared at (a) $\mathrm{pH} 3$, (b) $\mathrm{pH} 5$, (c) $\mathrm{pH} 7$ and (d) $\mathrm{pH} 9$. The compacts were sintered at $1480^{\circ} \mathrm{C}$ for $4 \mathrm{~h}$. 
As shown in Fig. 2(b), particles within a given agglomerate unit undergo faster densification, generating a hard agglomerate unit which, in turn, induces a large interagglomerate second-generation pore by a bridging with other hard agglomerate units in the initial- and intermediate-stages of sintering.

Figure 3 shows sintered microstructures of $\mathrm{Al}_{2} \mathrm{O}_{3}$ specimens prepared by centrifugal casting of colloidal slips having various colloid/interface variables: (a) $\zeta=56 \mathrm{mV}, \log W=1.58, \rho_{\mathrm{g}}=66.9 \%, \mathrm{pH} 3$; (b) $\zeta=52 \mathrm{mV}, \log W=1.55, \rho_{\mathrm{g}}=48.3 \%, \mathrm{pH} 5$; (c) $\zeta=34 \mathrm{mV}, \log W=1.46, \rho_{\mathrm{g}}=36.4 \%, \mathrm{pH} \mathrm{7}$; (d) $\zeta=4$ $\mathrm{mV}, \log W=0, \rho_{\mathrm{g}}=31.4 \%, \mathrm{pH}$ 9. These specimens were heat-treated at $1480^{\circ} \mathrm{C}$ for $4 \mathrm{~h}$. Figure 3 clearly shows that colloid/interface variables $(\zeta, W, \mathrm{pH})$ greatly influence on the homogeneity of sintered microstructure and the grain-growth rate. The sample fabricated using the slip at $\mathrm{pH} 3$ is already in the final stage of sintering with $\rho=98.2 \%$ of theoretical density $\left(\rho_{\text {th }}\right)$ and shows appreciable grain growth.

Figure 4 shows densification behavior of $\mathrm{Al}_{2} \mathrm{O}_{3}$ compacts heat-treated at $1480^{\circ} \mathrm{C}$ as a function of soaking time. Green compacts were fabricated by centrifugal casting. The result clearly shows that specimens derived from kinetically stable slips (low $\mathrm{pH}$ ) attain higher density than those derived from kinetically unstable slips. Figure 4 also shows that all the samples fired at $1480^{\circ} \mathrm{C}$ for $8 \mathrm{~h}$ essentially attain their end-point density regardless of such fabricating condition as $\mathrm{pH}$. This indicates that the secondgeneration pores originated from the interagglomerate pores (Fig. 3(d)) are difficult to remove during sintering and the amount of these pores is predetermined by the colloid/interface variables of $\mathrm{Al}_{2} \mathrm{O}_{3}$ slip.

In Fig. 5, we show scanning electron micrographs of yttria-partially stabilized zirconia $\left(3 \mathrm{~mol} \% \mathrm{Y}_{2} \mathrm{O}_{3}\right)$ specimens sintered at $1550^{\circ} \mathrm{C}$. The specimen fabricated using a kinetically stable slip $(\mathrm{pH} 3, \zeta=40 \mathrm{mV}$, $\log W=1.21, \rho_{\mathrm{g}}=48 \%$ of $\rho_{\mathrm{th}}$ ) is mostly composed of regions of dense and homogeneous microstructure

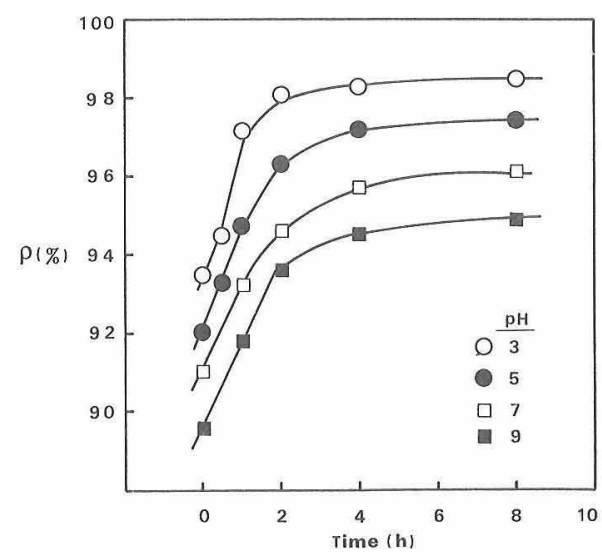

Fig. 4. Sintered density of alumina as a function of soaking time at $1480^{\circ} \mathrm{C}$. Green bodies were prepared at $\mathrm{pH}$ 's $3,5,7$ and 9.

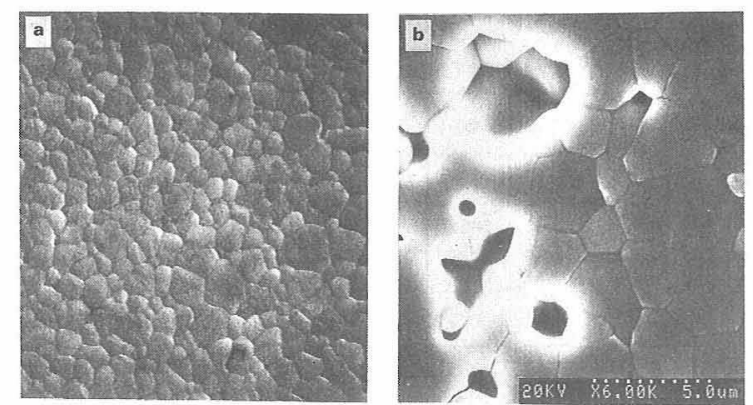

Fig. 5. Scanning electron micrographs of yttria-doped zirconia (3 mol\% yttria) specimens sintered at $1550^{\circ} \mathrm{C}$ (a) for $1 \mathrm{~h}$ and (b) for $8 \mathrm{~h}$. The conditions of fabrication of green bodies, (a) and (b), are described in the text.

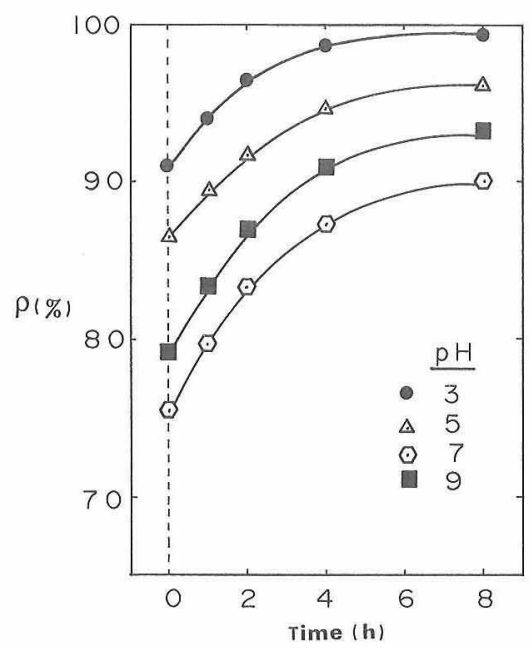

Fig. 6. Sintered density of yttria-doped zirconia as a function of sintering time at $1550^{\circ} \mathrm{C}$. Green bodies were prepared at $\mathrm{pH}$ 's 3,5 , 7 and 9.

$\left(1550^{\circ} \mathrm{C}\right.$ for $\left.1 \mathrm{~h}\right)$, as shown in Fig. 5 (a). On the other hand, the sample prepared under the condition of low kinetic stability ( $\mathrm{pH} 7, \zeta=-4 \mathrm{mV}, \log W=0, \rho_{\mathrm{g}}$ $=27 \%$ of $\rho_{\text {th }}$ ) shows large pores essentially unshrunken after sintering at $1550^{\circ} \mathrm{C}$ for $8 \mathrm{~h}$ (Fig. 5 (b)). Densification kinetics of yttria-partially stablized zirconia (Y-PSZ) is also shown in Fig. 6. The green compacts were fired at $1550^{\circ} \mathrm{C}$. The result again shows that specimens derived from kinetically stable slips (high $\zeta$ or $W$ ) attain much higher density than those derived from unstable slips for a fixed firing time.

Figure 7 shows the cumulative pore-size distribution of $\mathrm{Al}_{2} \mathrm{O}_{3}$ specimens after sintering to different temperatures. The green compacts were prepared using the kinetically unstable slips at $\mathrm{pH} 9 \quad(\zeta=4$ $\mathrm{mV}, \log W=0)$. From the pore-size distribution and the result of Fig. 3(d), it is seen that during sintering, the finer pores disappear rather rapidly but the larger interagglomerate (second-generation) pores essentially remain unchanged until the specimen practically reaches its end-point density. Although mercury-porosimetry data do not necessarily result 


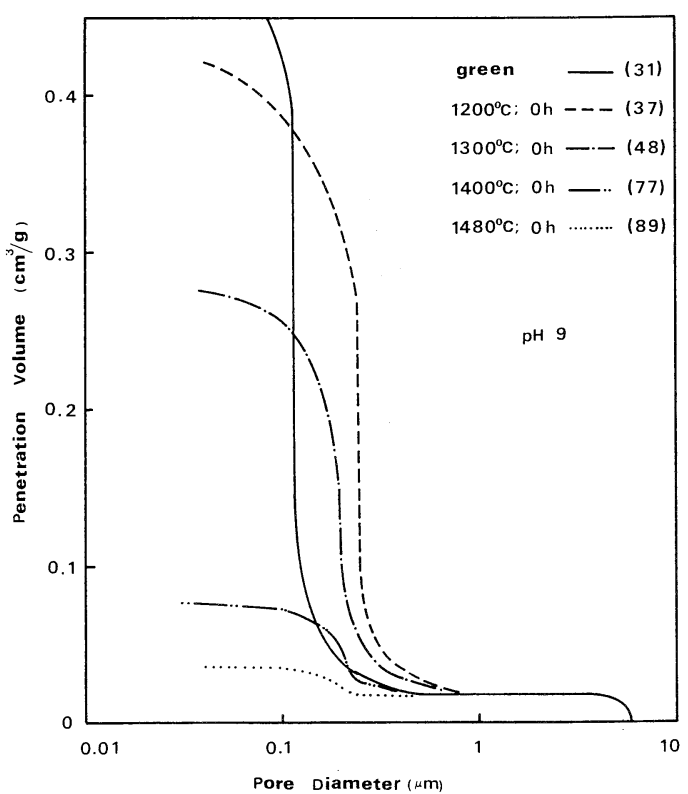

Fig. 7. Cumulative pore-size distribution of alumina specimens prepared at $\mathrm{pH} 9$ and sintered at various temperatures for $0 \mathrm{~h}$ (no holding time). The number in the parenthesis indicates relative density (percent of the theoretical density) of specimen.

in a correct pore-size distribution, the results support the previously made conclusion that the secondgeneration pores originated from the interagglomerate pores are difficult to remove. Figure 7 also shows that the most frequent pore diameter (abbreviated as MFPD hereafter) or the mean pore size increases and then decreases with increasing density. The cause of the observed pore-size increase at the beginning of densification is probably that the fine aggregates shrink and densify first, and this differential shrinkage contributes more space to the larger pores. The observation is consistent with previous investigations ${ }^{1,5), 13), 14)}$ of the pore-size evolution during the initial- and intermediate-stages of sintering.

The large second-generation pores are more difficult to remove for two reasons. These were summarized in a work done by Zhao and Harmer ${ }^{3}$ : First, as predicted by the scaling law, ${ }^{15)}$ it takes much longer time to fill a larger pore by diffusion. Second, as pointed out by Kingery and Francois, ${ }^{6}$ a large pore can be thermodynamically stable depending on the value of dihedral angle and the ratio of pore size to grain size. For a given dihedral angle and pore size there is a critical grain size below which the pore is stable. Because grain growth decreases the grain coordination number surrounding a large pore, thus making it thermodynamically unstable and sinterable, one can conclude that some grain growth is helpful to sintering. ${ }^{5), 16)}$ This creates a contradictory problem, however, because the kinetics of densification is adversely affected, to a considerable degree, by grain growth. ${ }^{3)}$ Zhao and Harmer showed that it is generally more favorable to inhibit grain growth than to promote it even though thermodynamics

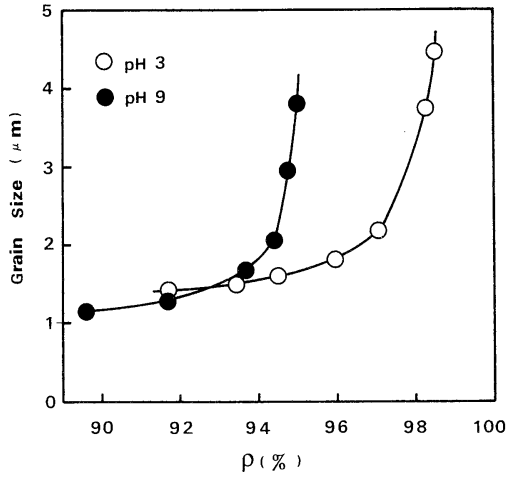

Fig. 8. Grain size of two alumina specimens (prepared at $\mathrm{pH}$ 's 3 and 9 and sintered at $1480^{\circ} \mathrm{C}$ ) as a function of relative density.

favors the grain growth for the shrinkage of large pores. Thus, the densification curves flattened out considerably at a longer time regime $(\sim 8 \mathrm{~h}$ in Figs. 4 and 6) reflect an inhibition to densification due to rapid grain growth at higher densities as well as a slow pore-shrinkage rate due to the interagglomerate second-generation pores.

In Fig. 8 we show the measured grain size/density trajectories for two different sets of $\mathrm{Al}_{2} \mathrm{O}_{3}$ samples prepared using aqueous slips of quite different kinetic stability. The compact derived from the suspension at $\mathrm{pH} 3$ densifies significantly $\left(\sim 97 \%\right.$ of $\left.\rho_{\text {th }}\right)$ before it exhibits an appreciable grain coarsening, whereas the sample prepared at $\mathrm{pH} 9$ shows an extensive grain growth before it reaches its end-point density $\left(\sim 95 \%\right.$ of $\left.\rho_{\text {th }}\right)$, where the matrix part essentially completes densification. The results shown in Figs. 4 and 8, thus, indicate that densely packed compact fabricated using a kinetically stable slip sinters to a higher density and to a finer grain size (at a given density) than a less densely packed compact. A comparison of the result of Fig. 8 with that of Fig. 4 also indicates that the onset of the rapid grain growth is directly related with the observed inflection point in the density versus time curve in Fig. 4 , that is, $\sim 98 \%$ and $\sim 94 \%$ of $\rho_{\text {th }}$ for $\mathrm{Al}_{2} \mathrm{O}_{3}$ compacts derived at $\mathrm{pH} 3$ and $\mathrm{pH} 9$, respectively.

3.2 Effects of green-body characteristics on pore elimination

In the previous section we have shown that the interagglomerate second-generation pores are difficult to remove and, for a given ceramic slip, colloid/interface variables essentially control densification behavior and microstructure development. In this section we focus on the effects of green body characteristics (density and pore size) on pore elimination and densification during sintering.

Since the rate of grain growth around a large pore is controlled by the pore mobility $\left(M_{\mathrm{p}}\right)$ rather than by the boundary mobility $\left(M_{\mathrm{b}}\right)$, the following equation can be written for the rate of grain growth around a large pore: ${ }^{3), 17)}$ 


$$
\frac{\mathrm{d} G}{\mathrm{~d} t}=\frac{2 M_{\mathrm{p}} F_{\mathrm{b}}}{n}
$$

and

$$
\begin{aligned}
& M_{\mathrm{p}}=\frac{D_{\mathrm{s}} \delta_{\mathrm{s}} \Omega}{k T \pi r^{4}} \quad \text { (for surface diffusion) or } \\
& M_{\mathrm{p}}=\frac{D_{1} \Omega}{k T \pi r^{3}} \quad \text { (for lattice diffusion) }
\end{aligned}
$$

where $F_{\mathrm{b}}$ is the force acting on the grain boundary, $M_{\mathrm{p}}$ is the pore mobility, $n$ is the number of pores per unit boundary area, $D_{\mathrm{s}}$ and $D_{1}$ are the surface diffusion coefficient and the lattice diffusion coefficient, respectively, $\delta_{\mathrm{s}}$ is the diffusion width, $\Omega$ is the atomic volume, and $r$ is the pore size. The above equation predicts that the grain-growth rate around a large pore is significantly retarded due to a small value of pore mobility $\left(M_{\mathrm{p}}-r^{-n}\right.$, where $n=3$ or 4 .). Therefore, it is rather difficult for a grain around a large pore to reach the critical size above which the pore is unstable.

If pores smaller than a certain critical size (or critical coordination number, $R_{\mathrm{c}}$ ) can be eliminated by enough time of sintering, the pore volume per unit weight of sample that can disappear during sintering can be formally written as ${ }^{5)}$

$$
V_{\mathrm{T}}=\int_{4}^{R_{\mathrm{c}}} V_{\mathrm{p}}(R) \mathrm{d} R
$$

where $V_{\mathrm{p}}(R)$ is the pore volume (per unit weight of specimen) associated with the coordination number $R$, and the lowest coordination number is assumed to be 4 . Since it is not possible to estimate the coordination-number distribution except for ideal packing geometries, we have derived an alternative expression for $V_{\mathrm{T}}$ in terms of measurable quantities, namely,

$$
V_{\mathrm{T}}=\frac{\rho(\infty)-\rho_{\mathrm{g}}}{\rho(\infty) \rho_{\mathrm{g}}}
$$

where $\rho(\infty)$ is the density of the end-point where the matrix part (excluding the volume occupied by the second-generation pores) fully densifies, and $\rho_{\mathrm{g}}$ is the density of green body. In the derivation of the above equation we assumed that pores larger than a certain critical size (or with the coordination number higher than $R_{\mathrm{c}}$ ) do not disappear unitil the compact practically reaches its end-point density. The pore volume per unit weight of sample that can be eliminated $\left(V_{\mathrm{T}}\right)$ was calculated using Eq. (4) and the data shown in Fig. 4, and the results for four different $\mathrm{Al}_{2} \mathrm{O}_{3}$ specimens fabricated using different $\mathrm{pH}$ conditions are shown in Table 1 . Using these results, that is, $V_{\mathrm{T}}$, and the pore-size distribution data for $\mathrm{Al}_{2} \mathrm{O}_{3}$ green compacts, one can estimate the critical size above which a complete elimination of pores is difficult. In this estimation we assumed that the finest pores disappear first but the larger pores (second-generation) shrink very slowly due to the
Table 1. Estimated Removable Pore Volume $\left(V_{\mathrm{T}}\right)$ of Alumina Samples (Per Weight) Prepared at Four Different pH Conditions

\begin{tabular}{|c|c|c|c|}
\hline $\mathrm{pH}$ & $\rho_{\mathrm{g}}\left(\mathrm{gm} / \mathrm{cm}^{3}\right)$ & $\rho(\infty)\left(\mathrm{gm} / \mathrm{cm}^{3}\right)$ & $V_{\mathrm{T}}\left(\mathrm{cm}^{3} / \mathrm{gm}\right)$ \\
\hline 3 & 2.656 & 3.906 & 0.120 \\
\hline 5 & 1.918 & 3.867 & 0.263 \\
\hline 7 & 1.445 & 3.815 & 0.430 \\
\hline 9 & 1.247 & 3.772 & 0.537 \\
\hline
\end{tabular}

thermodynamic and kinetic reasons, as discussed extensively in this and the previous sections. The estimated critical pore size that can be removable is approximately $3-4 \mu \mathrm{m}$ and is shown in Fig. 9 together with the cumulative pore-size distribution curves of four $\mathrm{Al}_{2} \mathrm{O}_{3}$ green compacts prepared using different $\mathrm{pH}$ conditions. The estimated critical pore size is a typical size for the second-generation pore which is induced by a bridging of hard agglomerates of a few $\mu \mathrm{m}$ size during the initial stage of sintering.

In Fig. 10 the sintered density of $\mathrm{Al}_{2} \mathrm{O}_{3}$ is plotted as a function of the most frequent pore diameter (MFPD) of the corresponding green compacts. The result shows that the compact with smaller MFPD consistently exhibits higher density than the compact with larger MFPD throughout sintering time. The fact that the sintered density is inversely proportional to the MFPD indicates an important role of green microstructure on sintering behavior and indireclty shows a decisive role of the colloid stability of ceramic slip on densification kinetics. An inverse linear relationship between the green density and the MFPD can be predicted based on the results shown in Figs. 9 (green density) and 10, and this type of correlation was demonstrated by other investigators. ${ }^{18), 19)}$

\subsection{Analysis of densification kinetics}

In the densification of porous polycrystalline compacts the matrix part, consisting of polycrystalline

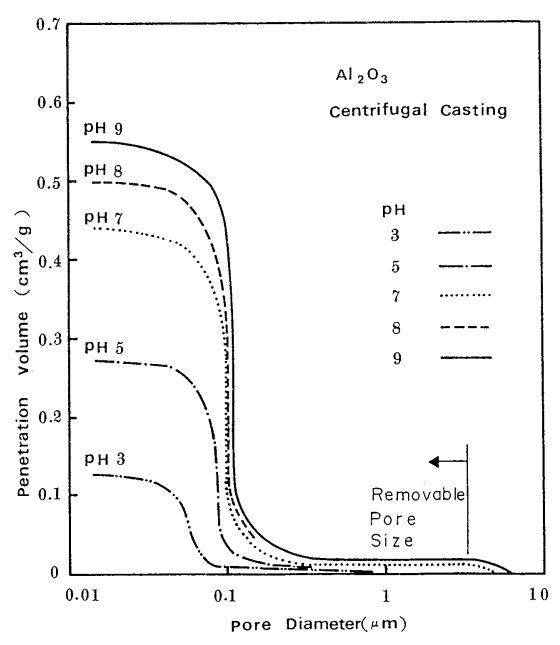

Fig. 9. Cumulative pore-size distribution of alumina green bodies prepared at various $\mathrm{pH}$ conditions. The figure also shows the upper limit of removable pore size during sintering. 


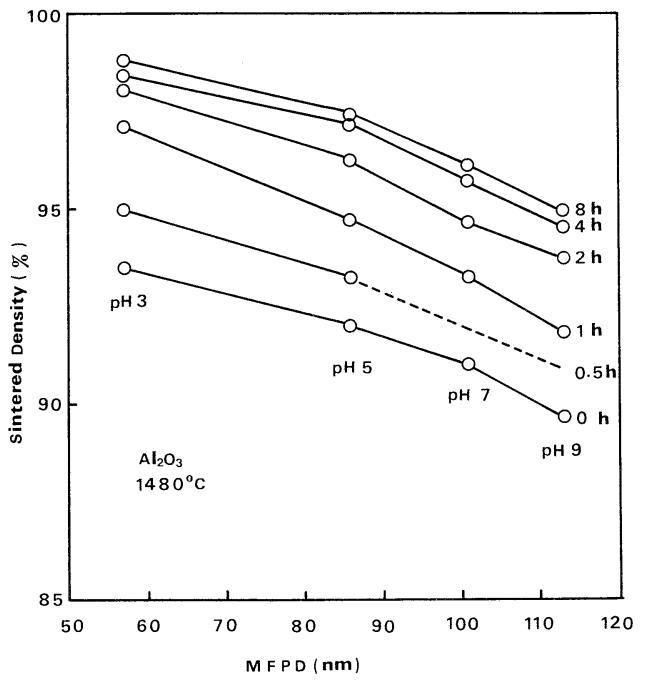

Fig. 10. Sintered density of alumina for various sintering times at $1480^{\circ} \mathrm{C}$ as a function of the most frequent pore diameter of the corresponding green bodies.

solid grains and the first-generation type of the fine matrix pores, usually densifies first, and the larger second-generation pores essentially remain unchanged until the matrix part fully densifies (Figs. 3, 5 and 7). In addition to this adverse densification effect due to the second-generation pores, there is a competing effect of densification and grain growth.

To understand the time evolution of the relative density, $\rho(t)$, one should find an adequate expression for the densification kinetics of the matrix part, $\rho_{\mathrm{m}}(t)$. To do this, we first considered Coble's wellknown theory of the intermediate and final stages of densification for tetrakaidecahedron grains: ${ }^{20)}$

$$
\frac{\mathrm{d} \rho_{\mathrm{m}}}{\mathrm{d} t}=\frac{\beta D_{1} \gamma_{\mathrm{s}} \Omega}{k_{\mathrm{B}} T[G(t)]^{3}}
$$

where $D_{\mathrm{l}}$ is the lattice diffusion coefficient, $\gamma_{\mathrm{s}}$ is the solid/vapor surface energy, $\Omega$ is the atomic volume, $k_{\mathrm{B}}$ is the Boltzmann' constant, and $G(t)$ is the grain size of the polyhedron at time $t$ for an isothermal sintering. $\beta$ is $160 \sqrt{2}$ for the cylindrical pore (for the intermediate stage of sintering) and $96 \pi$ for the spherical pore (for the final stage of sintering).

Since an accurate analytical derivation of $G(t)$ for the matrix part is extremely difficult task, we have employed a semiempirical relationship for $G(t)$ :

$$
G^{m}=G_{0}{ }^{m}+\alpha t
$$

where $t$ is sintering time at a given temperature, $G_{0}$ is the grain size at $t=0$, and $m$ is a constant usually lying between 1 and 3 . Early theories of grain growth were based on the following three basic assumptions: (i) The mobility and surface energy of all grain boundaries are equal; (ii) The grain-size distribution is time-invariant; (iii) The driving force for grain-growth process is proportional to $1 / G$, where $G$ is the average grain size. With these assumptions it can be shown that grain growth should obey the above equation with $m=2$. A more detailed discussion of the grain growth during sintering is given, for example, by Lay. ${ }^{21)}$

Combining Eqs. (5) and (6) and rearranging them yields an expression for $\rho_{\mathrm{m}}(t)$ during the early stage of an isothermal soaking experiment (early stage of grain growth):

where

$$
\left[1-\rho_{\mathrm{m}}(t)\right]=\left[1-\rho_{\mathrm{m}}(0)\right] \exp (-k t)
$$

$$
k=\frac{\beta D_{1} \gamma_{\mathrm{s}} \Omega}{k_{\mathrm{B}} T G_{0}{ }^{3}\left[1-\rho_{\mathrm{m}}(0)\right]}
$$

The above equation is valid for $G(t)<2^{1 / m} G_{0}$.

Since the large second-generation pores essentially remain unchanged during the early stage of grain growth, the relative density of polycrystalline body, $\rho(t)$, can be written in terms of the time-dependent density for the matrix part, $\rho_{\mathrm{m}}(t)$, namely,

$$
\rho_{\mathrm{t}}=\frac{V_{\mathrm{s}} \rho_{\mathrm{m}}(t)}{V_{\mathrm{s}}+V_{1} \rho_{\mathrm{m}}(t)}
$$

where $V_{\mathrm{s}}$ is the volume occupied exclusively by a polycrystalline solid having unit weight $\left(0.252 \mathrm{~cm}^{3} /\right.$ $\mathrm{g}$ for $\mathrm{Al}_{2} \mathrm{O}_{3}$ ), and $V_{1}$ is the volume occupied by the large second-generation pores originated from the interagglomerate pores in a green body. As shown in Fig. 9, the volume of the large interagglomerate pores per unit weight of $\mathrm{Al}_{2} \mathrm{O}_{3}$ compacts is approximately $0.01-0.015 \mathrm{~cm}^{3} / \mathrm{g}$. Therefore, for the $\mathrm{Al}_{2} \mathrm{O}_{3}$ compacts $V_{1}<(1 / 17) V_{\mathrm{s}}$. Under this condition, $\rho(t)$ in Eq. (8) can be approximated as $\rho_{\mathrm{m}}(t)$. Then, Eq. (7) suggests that the following linear equation approximately describes the densification kinetics of a porous polycrystalline body during the early stage of grain growth, where $G(t)<2^{1 / m} G_{0}$.

$$
P(t)=[1-\rho(t)]=[1-\rho(0)] \exp (-k t)
$$

where $P(t)$ is the porosity of polycrystalline specimen at time $t$. According to the above relationship the kinetics of pore shrinkage during the early stage of grain growth can be viewed as a first-order linear process with respect to the remaining pore volume during densification.

In Fig. 11 we show $\ln \{(1-\rho(t)) /(1-\rho(0))\}$ versus $t$ to for the $\mathrm{Al}_{2} \mathrm{O}_{3}$ compacts using the densification data shown in Fig. 4. As shown in the figure, the linear densification approximation (Eq. (9)) holds up to $\sim 2 \mathrm{~h}$ at $1480^{\circ} \mathrm{C}$. The rate constant of densification for the linear dinsification regime can be estimated from the result shown in Fig. 11 since the slope of the linear region is $-k$ (Eq. (9)). The estimated densification constants for the $\mathrm{Al}_{2} \mathrm{O}_{3}$ specimens prepared at several different $\mathrm{pH}$ conditions are as follows: $k=0.643 / \mathrm{h}(\mathrm{pH} 3) ; 0.380 / \mathrm{h}(\mathrm{pH} 5) ; 0.266 / \mathrm{h}$ $(\mathrm{pH} 7) ; 0.240 / \mathrm{h}(\mathrm{pH} 9)$. The result indicates that increasing the kinetic stability of ceramic slip ultimately enhances the rate constant for the linear densification regime. When the sintered density is sufficiently high, the competing effect of grain growth interferes 


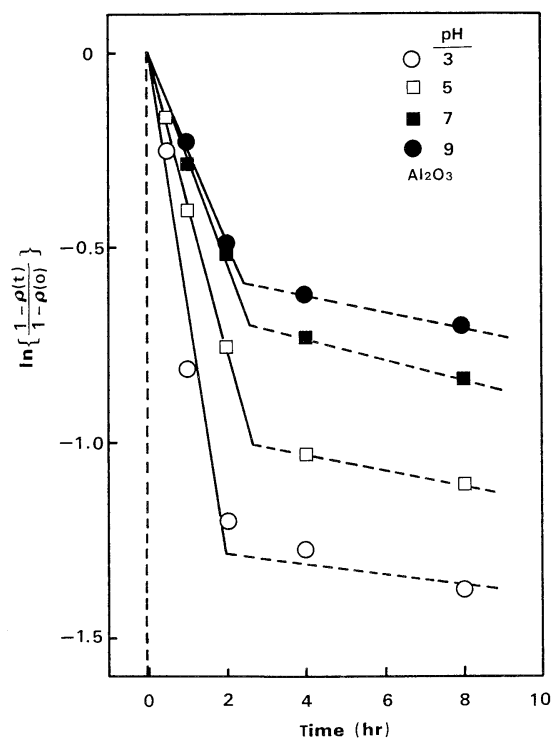

Fig. 11. Estimate of the rate constant $(k)$ of alumina for the linear densification regime.

further densification. In this case, the linear densification approximation (Eq. (9)) does not adequately describe the densification kinetics. Therefore, the inflection behaviors shown in Figs. 4 and $11(t=2-$ $3 \mathrm{~h}$ ) reflect the effects of grain growth and slow shrinkage kinetics of the second-generation pores on the densification rate of $\mathrm{Al}_{2} \mathrm{O}_{3}$.

Similarly, the linear densifcation-rate constants of Y-PSZ compacts $(3 \mathrm{~mol} \%) \mathrm{Y}_{2} \mathrm{O}_{3}$ can be estimated using Eq. (9) and the densification data shown in Fig. 6. As shown in Fig. 12, the linear densification approximation holds up to $4 \mathrm{~h}$ at $1550^{\circ} \mathrm{C}$. The estimated densifcation constants for the Y-PSZ compacts prepared at several different $\mathrm{pH}$ conditions are: $k=0.476 / \mathrm{h}(\mathrm{pH} 3, \zeta=40 \mathrm{mV}, \log W=1.21) ; 0.225 /$ h $(\mathrm{pH} 5, \zeta=33 \mathrm{mV}, \log W=0.94) ; 0.206 / \mathrm{h}(\mathrm{pH} 9$, $\zeta=-29 \mathrm{mV}, \log W=0.77) ; 0.156 / \mathrm{h}(\mathrm{pH} 7, \zeta=-4$ $\mathrm{mV}, \log W=0)$. The result demonstrates that the ki-

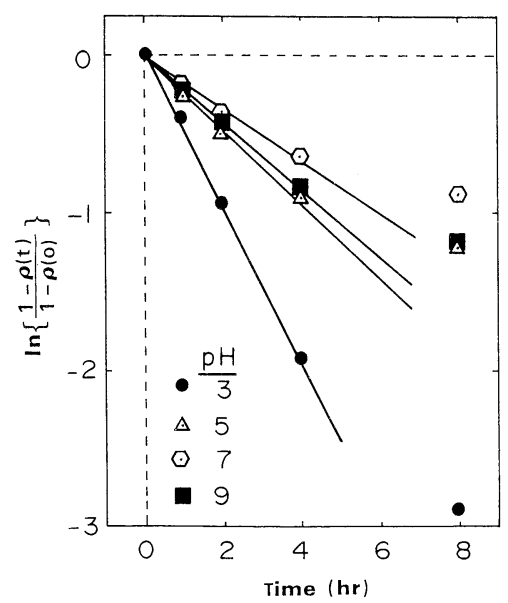

Fig. 12. Estimate of the rate constant $(k)$ of yttria-doped zirconia for the linear densification regime. netic stability of Y-PSZ slip also controls the densification rate.

\subsection{Correlation of colloidal stability with den-} sification kinetics

Figure 13 shows the green density $\left(\rho_{\mathrm{g}}\right)$ and the relative density of the $\mathrm{Al}_{2} \mathrm{O}_{3}$ compact at $t=0(\rho(0))$ as a function of the inverse of the linear densification-rate constant at $1480^{\circ} \mathrm{C}$. The result clearly demonstrates that increasing density of the green compact $\left(\rho_{\mathrm{g}}\right)$ of $\mathrm{Al}_{2} \mathrm{O}_{3}$ by increasing the kinetic stability of colloid suspension (high $W$ and $\zeta$ ) significantly enhances the linear densification-rate constant, $k$. As shown in Eq. (7), the density at $t=0$ for a given soaking temperature can be approximated as

$$
\rho(0)=1-\frac{\gamma}{G_{0}^{3}}\left(\frac{1}{k}\right)
$$

where

$$
\gamma=\frac{\beta D_{1} \gamma_{\mathrm{s}} \Omega}{k_{\mathrm{B}} T}
$$

Since $G_{0}$ and $\gamma$ for an isothermal sintering are constant, this equation justifies the observed linear relationship between $\rho(0)$ and $1 / k$ with the intercept being 1.0. From the slope $(S)$ of Fig. 13, one can estimate the lattice diffusion constant of the species relevant to the densification, namely,

$$
D_{1}=\frac{-k_{\mathrm{B}} T G_{0}{ }^{3} S}{\beta \gamma_{\mathrm{s}} \Omega}=1.2 \times 10^{-16} \mathrm{~m}^{2} \cdot \mathrm{s}^{-1}
$$

The values used in the estimate of $D_{1}$ are: $G_{0}=1.5$ $\mu \mathrm{m} ; \quad S=-4.2 \times 10^{-6} \mathrm{~s}^{-1} ; \quad \beta=300$ (for spherical pores); $\gamma_{\mathrm{s}}=0.9 \mathrm{Jm}^{-2} ;{ }^{2)} \Omega=2.1 \times 10^{-29} \mathrm{~m}^{3}{ }^{2)}$ The estimated $D_{1}$ is approximately consistent with the lattice diffusion coefficient of $\mathrm{Al}$ atom in $\mathrm{Al}_{2} \mathrm{O}_{3}$ at $1500^{\circ} \mathrm{C} .{ }^{22}$ )

As discussed extensively elsewhere, ${ }^{9), 11)}$ the overall kinetic stability of a given colloidal dispersion is determined by the stability ratio which is approximately defined as $^{23)}$

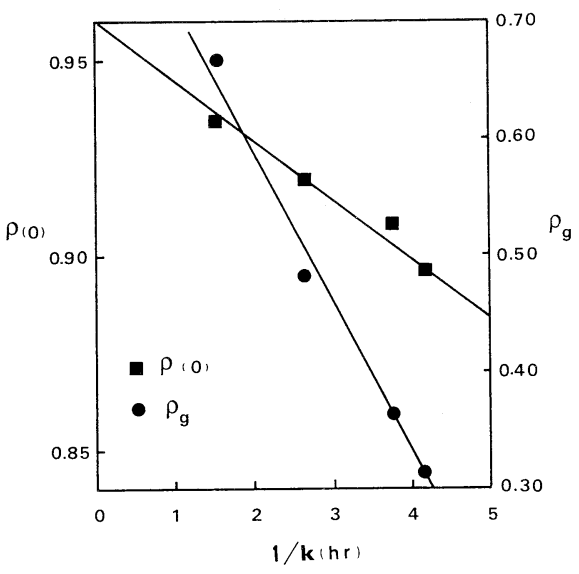

Fig. 13. Green density $\left(\rho_{\mathrm{g}}\right)$ and the density of compact at $t=0$ $(\rho(0))$ as a function of the reciprocal linear densification-rate constant of alumina. 
$W=$

total number of particle collisions for a fixed time interval number of collisions forming permanent flocculates

Figure 14 shows the packing density of $\mathrm{Al}_{2} \mathrm{O}_{3}$ (centrifugal casting) as a function of the stability ratio or the zeta potential. As shown in the previous paper, ${ }^{24)}$ the stability ratio or the zeta potential of $\mathrm{Al}_{2} \mathrm{O}_{3}$ suspension should be larger than a certain critical value for a green body with high packing density. The critical stability ratio $\left(W_{\mathrm{c}}\right)$ and the critical zeta potential $\left(\zeta_{\mathrm{c}}\right)$ of the $\mathrm{Al}_{2} \mathrm{O}_{3}$ suspension are 28 and $53 \mathrm{mV}$, respectively (Fig. 14).

The concept of the critical stability can also be correlated with densification kinetics. To do this we assumed that $\rho_{\mathrm{g}}$ is proportional to the inverse of the linear densification-rate constant $(1 / k)$. Experimental result shown in Fig. 13 clearly supports this assumption. Since the densification rate constant for a hypothetical single crystalline body $\left(\rho_{\mathrm{g}}=1\right)$ approaches to $\infty, \rho_{\mathrm{g}}$ can be semiempirically written as

$$
\rho_{\mathrm{g}}=1-S_{\mathrm{g}}\left(\frac{1}{k}\right)
$$

where $S_{\mathrm{g}}$ is the linear slope shown in Fig. 13. From the result of Fig. 14, $\rho_{\mathrm{g}}$ of $\mathrm{Al}_{2} \mathrm{O}_{3}$ compacts can be empirically expressed as

$$
\rho_{g}=a+b \log W
$$

where $a$ and $b$ are empirical constants (can be deduced from Fig. 14) having different characteristic values below and above the critical stability ratio, that is, $a=0.32, b=0.014$ below the critical point and $a=-3.124, b=2.371$ above the critical point. Combining Eq. (11) with Eq. (12) yields a semiempirical equation directly relating the densification rate with the colloidal stability, namely,

$$
\log W=\left(\frac{1-a}{b}\right)-\left(\frac{S_{g}}{b}\right)\left(\frac{1}{k}\right)
$$

Thus, a plotting $\log W$ versus $1 / k$ yields a straight

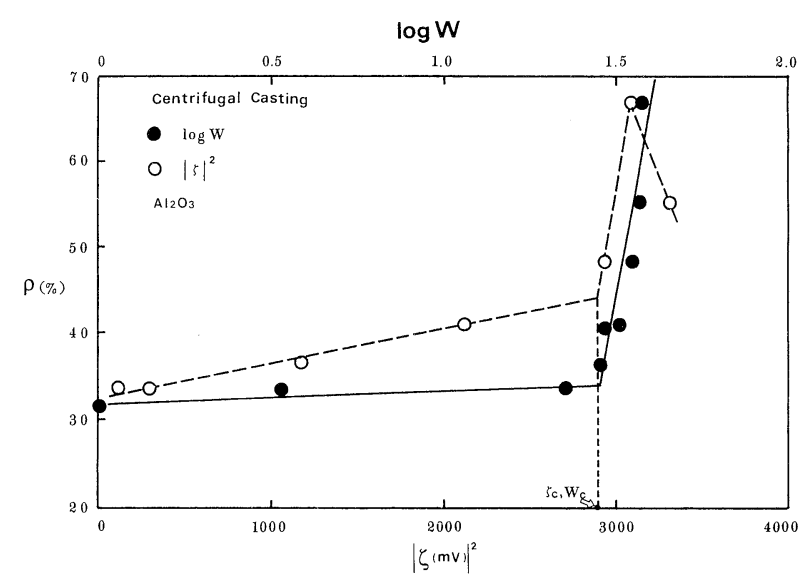

Fig: 14. Correlation of green density of alumina (prepared by centrifugal casting) with $\log W$ and $|\zeta|^{2}$ of suspension. line with a slope being $-S_{\mathrm{g}} / b$. Similar equation can be obtained for $|\zeta|^{2}$ versus $1 / k$. Figure 15 shows a rapid decrease in the absolute value of the slope above the transition point, and the values of $W_{\mathrm{c}}$ and $\zeta_{c}$ estimated from Fig. 15 are exactly consistent with those obtained from the analysis of $\rho_{\mathrm{g}}$ versus $\log W$ or $|\zeta|^{2}$, as shown in Fig. 14. The observed value of the intercept for $\log W$ versus $1 / k$ plot in Fig. 15 is 1.75 , and this value accords well with that of semiempirical prediction in Eq. (13), that is, $(1-a) /$ $b=1.74$. As shown in Fig. 16, essentially the same type of correlation was obtained for the Y-PSZ system. Therefore, the results of Fig. 15 and Fig. 16 directly demonstrate important roles of the colloid/ interface variables (colloidal stability) on the densification kinetics of polycrystalline ceramics.

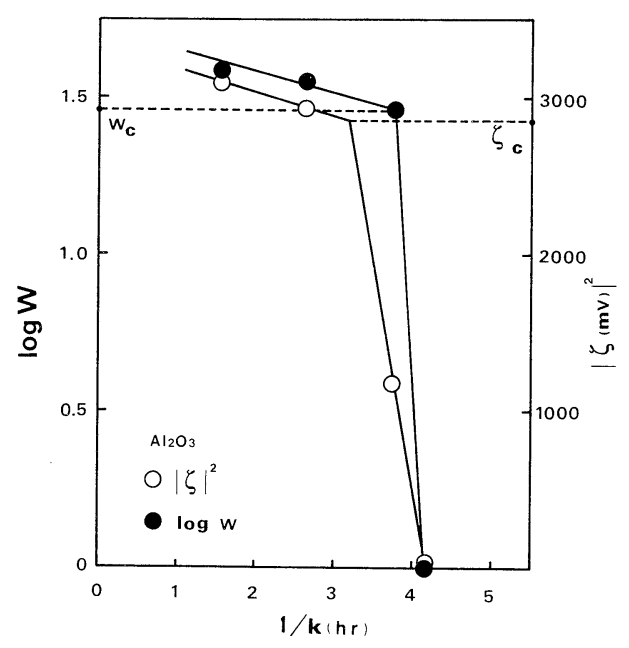

Fig. 15. Correlation of $\log W$ and $|\zeta|^{2}$ of suspension with the inverse of the linear densification-rate constant of alumina.

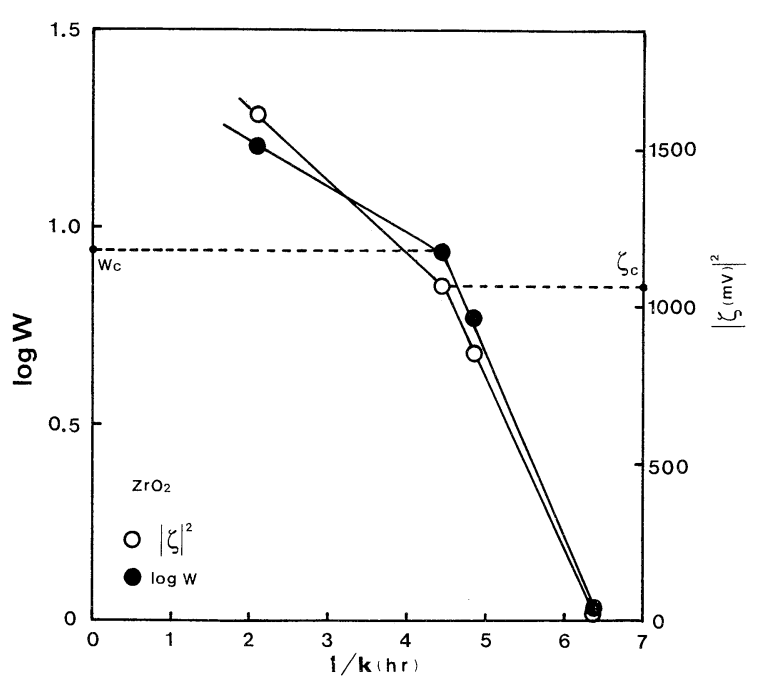

Fig. 16. Correlation of $\log W$ and $|\zeta|^{2}$ of suspension with the inverse of the linear densification-rate constant of yttria-doped zirconia. 


\section{Conclusions}

Effects of colloid/interface variables on the densification kinetics were examined using $\mathrm{Al}_{2} \mathrm{O}_{3}$ and $\mathrm{Y}$ PSZ as model systems. Both $\mathrm{Al}_{2} \mathrm{O}_{3}$ and $\mathrm{Y}-\mathrm{PSZ}$ specimens fabricated using kinetically stable colloidal suspensions (high $W$ and $\zeta$ ) exhibited dense and homogeneous microstructures and faster densification rates. On the other hand, specimens prepared under the condition of low kinetic stability showed large pores unshrunken even after prolonged sintering.

Using isothermal densifcation data and pore-size distribution of $\mathrm{Al}_{2} \mathrm{O}_{3}$ green compacts, we have estimated the critical size above which a complete elimination of pores is difficult. The estimated critical pore size $(3-4 \mu \mathrm{m})$ is a typical size for the secondgeneration pore which is induced by a bridging of hard agglomerates of a few $\mu \mathrm{m}$ size during the initial stage of sintering.

Analysis of the densification data showed that increasing the kinetic stability of suspension significantly enhances the rate constant of sintering for the linear densification regime (early stage of grain growth). A concept of the critical stability of colloidal suspension was applied to the densification $\mathrm{ki}$ netics of $\mathrm{Al}_{2} \mathrm{O}_{3}$ and Y-PSZ. This demonstrated a direct linear correlation of the stability ratio $(W)$ with the inverse of the densification-rate constant $(1 / k)$ with a transition behavior at the critical stability point $\left(W_{\mathrm{c}}\right.$ or $\left.\zeta_{\mathrm{c}}\right)$.

Acknowledgments We gratefully acknowledge the financial support provided by the Korea Science and Engineering Foundation (KOSEF) through the Center for Interface Science and Engineering of Materials.

\section{References}

1) J. Zheng and J. S. Reed, J. Am. Ceram. Soc., 72, 810-17
(1989).

2) J. Zhao and M. P. Harmer, J. Am. Ceram. Soc., 71, 113-20 (1988).

3) J. Zhao and M. P. Harmer, J. Am. Ceram. Soc., 71, 530-39 (1988).

4) E. A. Barringer and H. K. Bowen, J. Am. Ceram. Soc., 62, C199-201 (1982).

5) F. F. Lange, J. Am. Ceram. Soc., 67, 83-88 (1984).

6) W. D. Kingery and B. Francois, "Sintering and Related Phenomena”, Ed. by G. C. Kuczynski, N. A. Hooton and C. F. Gibbon, Gordon and Breach Science Publishers, New York (1967) pp. 471-96.

7) I. A. Aksay, "Advances in Ceramics, Vol. 9, Forming of Ceramics”, Ed. by J. A. Mangles, American Ceramic Society, Columbus, OH (1984) pp. 94-104.

8) F. F. Lange, J. Am. Ceram. Soc., 72, 3-15 (1989).

9) H. M. Jang and J. H. Moon, J. Mater. Res., 5, 615-22 (1990).

10) P. H. Wiersema, A. L. Loeb and J. Th. G. Overbeek, J. Colloid Interf. Sci., 22, 78-99 (1966).

11) J. Th. G. Overbeek, J. Colloid Interf. Sci., 58, 431-45 (1977).

12) M. I. Mendelson, J. Am. Ceram. Soc., 52, 443-46 (1969).

13) O. J. Whittemore and J. A. Valela, "Sintering Processes", Ed. by G. C. Kuczynski, Plenum Press, New York (1981) pp. 51-60.

14) T. Kimura, Y. Matsuda, M. Oda and T. Yamaguchi, Ceram. Int., 13, 27-34 (1987).

15) C. Herring, J. Appl. Phys., 21, 301-03 (1950).

16) F. F. Lange and B. J. Kellett, J. Am. Ceram. Soc., 72, 735-41 (1989).

17) R. J. Brook, J. Am. Ceram. Soc.,52, 56-57 (1969).

18) R. G. Frey and J. W. Halloran, J. Am. Ceram. Soc.,67, 199203 (1984).

19) A. Roosen and H. K. Bowen, J. Am. Ceram. Soc.,71, 970-77 (1988).

20) R. L. Coble, J. Appl. Phys., 32, 787-92 (1961).

21) K. W. Lay, "Sintering and Related Phenomena", Ed. by G. C. Kuczynski, Plenum Press, New York (1973) pp. 65-80.

22) W. D. Kingery, H. K. Bowen and D. R. Uhlmann, "Introduction to Ceramics", 2nd ed., John Wiley \& Sons, New York (1976) p. 240.

23) R. J. Hunter, "Zeta Potential in Colloid Science, Principles and Applications", Academic Press (1981) Chapter 6.

24) H. M. Jang, J. H. Moon and B. H. Kim, J. Ceram. Soc. Japan., 102, 119-27 (1994). 